Published in final edited form as:

Int J Antimicrob Agents. 2008 February ; 31(Supplement 1): 112-116.

\title{
Treatment of chronic prostatitis/chronic pelvic pain syndrome
}

\author{
J. Curtis Nickel ${ }^{*}$ \\ Department of Urology, Queen's University, Kingston General Hospital, Kingston, Ontario, Canada \\ $K 7 L 2 V 7$
}

\begin{abstract}
Acceptance of the National Institutes of Health definition of Category III Chronic Prostatitis/Chronic Pelvic Pain Syndrome (CP/CPPS) and the development and validation of the Chronic Prostatitis Symptom Index has stimulated significant research into treatment of this condition. Evidence-based suggestions for treatment include the following. (i) Antimicrobials cannot be recommended for men with longstanding, previously treated CP/CPPS. (ii) Alpha-blockers can be recommended as firstline medical therapy, particularly in alpha-blocker-naïve men with moderately severe symptoms who have relatively recent onset of symptoms. (iii) Alpha-blockers cannot be recommended in men with longstanding CP/CPPS who have tried and failed alpha-blockers in the past. And (iv) antiinflammatory therapy, finasteride and pentosan polysulfate are not recommended as primary treatment; however, they may have a useful adjunctive role in a multimodal therapeutic regimen. Early data on herbal therapies, particularly quercetin and cernilton, are intriguing, but larger multicentre, randomised, placebo-controlled trials are required before a high level of evidence recommendation can be made on its use. At this time, surgery (including minimally invasive) is recommended only for definitive indications and not generally for CP/CPPS.
\end{abstract}

\section{Keywords}

Prostatitis; Chronic pelvic pain syndrome; Treatment

\section{Introduction}

The majority of men diagnosed with prostatitis do not have a demonstrable bacterial aetiology to explain their symptom complex. However, for decades almost all the literature with regard to treatment, and even the standard of practice, involved antimicrobial therapy for anyone with a diagnosis of prostatitis. The academic research community almost completely ignored the common, but enigmatic, conditions of non-bacterial prostatitis and prostatodynia. Once bacterial infection of the prostate was ruled out and/or the patient did not respond to antimicrobial therapy, the practicing physician did not know how to proceed in terms of management. There were no evidence-based guidelines or even reasonable advice on how to treat these patients. The National Institutes of Health (NIH) Consensus Conference (Bethesda, VA) in 1995 initiated a field change in our attitude regarding prostatitis and changed the direction of clinical research in the prostatitis field. A major advance was to define the group

\footnotetext{
* Tel.: +1 613372 0501; fax: +1 613545 1970., E-mail address: jen@ post.queensu.ca.

Competing interests: J.C.N. is a consultant for Merck, GlaxoSmithKline, sanofiaventis, Ortho-McNeil, Farr Laboratories and Triton Pharma Inc., and an investigator for Merck, GlaxoSmithKline, sanofi-aventis and Ortho-McNeil.

Ethical approval: Not required.

Publisher's Disclaimer: This is a PDF file of an unedited manuscript that has been accepted for publication. As a service to our customers we are providing this early version of the manuscript. The manuscript will undergo copyediting, typesetting, and review of the resulting proof before it is published in its final citable form. Please note that during the production process errors may be discovered which could affect the content, and all legal disclaimers that apply to the journal pertain.
} 
of patients with chronic pelvic pain but no infection (detected by standard medical microbiological) as Category III Chronic Prostatitis/Chronic Pelvic Pain Syndrome (CP/CPPS) [1]. The next major advance was the development and validation of an outcome measure, the NIH Chronic Prostatitis Symptom Index (NIH-CPSI) [2], a tool that was greatly needed to evaluate symptoms in clinical trials. The NIH spearheaded clinical treatment studies, whilst the pharmaceutical and medical industry realised the market potential and followed with studies of their own. The plethora of data on treatment of CP/CPPS over the last 5-10 years now allows us to provide an evidence-based management plan for patients diagnosed with CP/CPPS.

\section{Traditional therapy}

Treatment for CP/CPPS has been traditionally based on our concept of 'prostatitis' and therefore centred on infection and inflammation of the prostate. It is no wonder that the most common therapies were antibiotics and anti-inflammatories. Because we had become so familiar with medical therapy for that other benign prostatic condition, benign prostatic hyperplasia, it seemed natural to treat 'prostatitis' with alpha-blockers, 5-alpha reductase inhibitors, prostate-related phytotherapy and, for desperate patients, prostate surgery (invasive and minimally invasive). Pain was managed with anti-inflammatories, antianxiolytics, analgesics (narcotics and other), antidepressants and, again for desperate patients, surgery. However, except for some small uncontrolled studies with unclear enrolment protocols and non-validated outcomes, there was absolutely no proof that any of these approaches were clinically efficacious for the patient with a diagnosis of prostatitis not related to a demonstrable infectious aetiology.

\section{What has changed our understanding of CP/CPPS therapy?}

Standardisation of the definition and classification of the patient presenting with a prostatitis diagnosis as well as development and validation of the NIH-CPSI [2,3] have been a major stimuli to the design and completion of an increasing number of well designed treatment trials. The NIH-CPSI covers the three most important domains of a prostatitis patient's experience (pain scored from 0-21; voiding scored from 0-10; and impact on quality of life scored from $0-12$; total possible score 43 ). A decrease of 4-6 points or a $25 \%$ decrease in total score appears to be perceptible to a patient as a clinical improvement [3-6]. A difference of 3 between the change in NIH-CPSI score from baseline in a treatment group compared with the change in a placebo group (the 'treatment effect') appears to be a clinically significant effect when studying differences in groups in a randomised, placebo-controlled trial [3-6].

\section{What constitutes clinical evidence for treatment guidelines in CP/CPPS}

The author has suggested that evidence for treatment guidelines in CP/CPPS should come from clinical trials that conform to very strict criteria [7]:

a. NIH classification system for definition and characterisation of patients;

b. randomised, placebo-controlled design;

c. validated outcome parameters (such as the NIH-CPSI); and

d. peer-reviewed (published in peer-reviewed literature).

Table 1 compares the results of presently available published peer-reviewed, randomised, placebo-controlled trials employing the standardised clinical definition of CP/CPPS and employing the NIH-CPSI as an outcome parameter. 


\subsection{Antimicrobials}

Approximately $50 \%$ of patients with CP/CPPS can be expected to improve with fluoroquinolone therapy [21], and although the evidence has come from some prospective studies, it has only been in the last few years that randomised, placebo-controlled trials were undertaken and reported $[11,15]$. Patients with long-term CP/CPPS symptoms who had received multiple prior treatments (including antimicrobials) appear to have similar symptom response to 6 weeks of levofloxacin [11] or ciprofloxacin [15] (responder rate 33\% and 22\%, respectively), which did not differ significantly from the benefits obtained with placebo (31\% and $22 \%$, respectively). However, in patients who have been symptomatic for only a short duration (median 4 weeks) and not previously treated with antibiotics, one can obtain a response rate of up to $75 \%$ in patients who cultured non-uropathogenic bacteria (definition of CP/CPPS) [22]. The same was observed in patients who actually cultured typical uropathogens (definition of Category II Chronic Bacterial Prostatitis) [22].

\subsection{Alpha-blockers}

Three randomised, placebo-controlled trials evaluating tamsulosin [14], terazosin [12] and alfuzosin [13] showed a statistically and likely clinically significant treatment effect with these alpha-blockers. However, a well powered, NIH Chronic Prostatitis Collaborative Research Network (CPCRN) trial of 6 weeks of the alpha-blocker tamsulosin appears to provide no additional benefit to placebo when used in CP/CPPS patients who have had the condition longterm and who had been heavily pre-treated (including previous treatment with alpha-blockers) [13]. An analysis of these four trials would indicate that longer courses (more than 6 weeks) of alpha-blockers provide modest benefits when prescribed to alpha-blocker-naïve CP/CPPS patients with shorter duration of disease. This hypothesis is presently being tested by the NIHCPCRN in an ongoing randomised controlled trial (RCT) comparing the effects of 12 weeks of alfuzosin compared with placebo in recently diagnosed alpha-blocker-naïve CP/CPPS patients.

\subsection{Anti-inflammatories}

The only large, multicentre, randomised, placebo-controlled trial evaluating antiinflammatories compared 6 weeks of $25 \mathrm{mg}$ and $50 \mathrm{mg}$ rofecoxib with placebo [16]. Only highdose rofecoxib provided statistically, but only modest clinically, significant benefit compared with placebo treatment. A very small, single-centre study (17 patients in total) [23] showed no obvious benefits of zafirlukast, a leukotriene antagonist, compared with placebo on the symptoms of Category IIIA CP/CPPS.

\subsection{Hormones}

Finasteride has been shown in small, poorly controlled trials to have a possible effect in CP/ CPPS, but a small, randomised, placebo-controlled study did not suggest significant efficacy as monotherapy [18]. Whilst twice as many patients responded to 6 months of finasteride compared with placebo, the actual magnitude of improvement did not reach statistical significance. A small, single-centre, pilot study suggested that mepartricin, a drug that lowers prostatic oestrogen levels, may provide some benefits [19], but a larger, well designed, multicentre trial is necessary to confirm this.

\subsection{Phytotherapy}

Unsupervised herbal-based therapies have become very popular with CP/CPPS patients. Quercetin, a natural bioflavonoid, has been shown to provide a statistically and clinically significant benefit compared with placebo in a very small, single-centre, pilot study [20]. Two unpublished RCTs presented at the annual American Urological Association's meeting have suggested benefits with Serenoa repens, a saw palmetto berry extract [24], and with 
Cernilton ${ }^{\circledR}$ [25], a pollen extract popular in Europe. Before this evidence can be employed to support a recommendation for treatment, these trials must undergo peer-review by being published in a peer-reviewed journal. More patients were cured or improved following 6 months of treatment with bee pollen extract compared with placebo in a small, single-centre, published study [26], but unfortunately the recently accepted validated outcome measures used by most contemporary researchers were not employed in this study, making interpretation and comparison difficult. Large, multicentre, well designed RCTs that have undergone peer-review processes will be required before we can make strong recommendations regarding this complementary and alternative medical approach.

\subsection{Interstitial cystitis treatment}

Pentosan polysulfate has been used in patients with interstitial cystitis, another chronic pelvic pain condition that occurs primarily in women, and a RCT comparing high-dose (900 mg/day) pentosan polysulfate with placebo in men with CP/CPPS showed modest benefits in some men [17].

\subsection{Other medical therapies}

Antianxiolytics, antidepressants (particularly the tricyclic antidepressants) and neuromodulatory agents (gabapentin and pregabalin) are used to treat the pain and associated symptoms of CP/CPPS, but no firm evidence is available to allow an evidence-based recommendation to be made [8]. Use of narcotics (opioids) in the management of the chronic pain of treatment-refractory CP/CPPS remains controversial and the real risks have to be weighed against the potential benefits. A recent review outlined the pros and cons of the use of opioids for the chronic non-malignant neuropathic pain experienced by patients with longterm treatment refractory CP/CPPS [27].

\subsection{Invasive procedures}

Uncontrolled clinical series employing bladder neck surgery have noted benefits in some $\mathrm{CP} /$ CPPS patients with obstructive voiding symptoms and urodynamically demonstrated bladder neck obstruction [28]. Radical transurethral resection of the prostate (TURP) and open prostatectomy have been advocated for CP/CPPS based on a few anecdotal experiences, but there are absolutely no reliable data or experiences to substantiate a treatment effect. Instead, a harmful outcome could potentially result from such therapy. A small, singe-centre study showed that transurethral microwave thermotherapy (TUMT) provided more long-term benefit in patients with Category IIIA CP/CPPS than did SHAM control [29]. A recent case series employing TUMT suggested a benefit [30], but before this minimally invasive therapy (MIT) can be adopted, a large, multicentre, SHAM-controlled trial employing contemporary definition and outcome parameters is required. Similarly, transurethral needle ablation (TUNA) has also been suggested as a therapeutic MIT, but a small SHAM-controlled study did not suggest efficacy [31].

\subsection{Recommendations for treatment of CP/CPPS}

Based on the evidence of prospectively designed, randomised, placebo-controlled trials enrolling a well defined population of men diagnosed with CP/CPPS and employing a validated outcome (NIH-CPSI), a number of recommendations regarding management have been suggested [7-9]. The International Consultation on Prostate Disease met in Paris in June 2005 and a committee chaired by A.J. Schaeffer [USA] (committee members included R.U. Anderson [USA], J.N. Krieger [USA], B. Lobel [France], K. Naber [Germany], M. Nakagawa [Japan], J.C. Nickel [Canada], L. Nyberg [USA] and W. Weidner [Germany]) reviewed the current evidence on the management of CP/CPPS [10]. Suggested treatment recommendations are shown in Table 2. 


\section{The future of CP/CPPS management}

Our evolving understanding of the aetiology and mechanism involved in the pathogenesis of $\mathrm{CP} / \mathrm{CPPS}$ will open new avenues of research and novel targets for therapeutic trials. It appears that the process begins with some form of initiator (infection, trauma, dysfunctional voiding, allergy etc.) causing inflammation or neurogenic damage in and around the prostate (pelvic floor, bladder, perineum etc.). If not dealt with quickly, peripheral and then central sensitisation occurs. Traditional therapy has been directed at the initiators of the process, a plan that might work in recently diagnosed CP/CPPS patients in whom the process has not progressed to the truly chronic stage. In all cases (recent onset and chronic), recognised initiators must be treated (e.g. voiding dysfunction, infection) or avoided (e.g. bicycle seat, diet). In patients who have developed a chronic inflammatory state, immune modulation may provide benefit. Some form of neuromodulation will likely turn out to be the key to therapy in patients who evolve into a chronic neuropathic pain state. Patients who develop pelvic floor neuromuscular dysfunction may respond to targeted physiotherapy. Once central nervous system sensitisation occurs and the patient enters a chronic neuropathic state, then higher brain centres modulate pain and disability (depression, anxiety, coping mechanisms etc.) and cognitive behavioural therapies may be beneficial [32].

The NIH-CPCRN and Interstitial Cystitis Collaborative Research Networks (ICCRN) have initiated trials evaluating therapy in early-onset disease (alfuzosin, amitriptyline). Trials in more chronic disease include immunomodulation (mycophenolate mofetil $\mathrm{HCl}$ or CellCept ${ }^{\circledR}$ ), neuromodulation (amitriptyline and pregabalin), physiotherapy and cognitive behavioural treatment protocols. It is hoped that these trials will result in decreased symptoms, increased tolerability to chronic pain, decreased disability and/or improved quality of life in patients with CP/CPPS.

\section{Acknowledgements}

Funding: National Institutes of Health/NIDDK, Merck Frosst Canada, GlaxoSmithKline and sanofi-aventis.

\section{References}

1. Krieger JN, Nyberg L Jr, Nickel JC. NIH consensus definition and classification of prostatitis. JAMA 1999;282:236-7. [PubMed: 10422990]

2. Litwin MS, McNaughton-Collins M, Fowler FJ Jr, et al. The National Institutes of Health. Chronic Prostatitis Symptom Index: development and validation of a new outcome measure. Chronic Prostatitis Collaborative Research Network. J Urol 1999;162:369-75. [PubMed: 10411041]

3. Propert KJ, Litwin M, Wang Y, et al. Responsiveness of the National Institutes of Health. Chronic Prostatitis Symptom Index (NIH-CPSI). Qual Life Res 2006;15:299-305. [PubMed: 16468084]

4. Turner JA, Ciol MA, Von Korff M, Berger R. Validity and responsiveness of the National Institutes Of Health Chronic Prostatitis Symptom Index. J Urol 2003;169:580-3. [PubMed: 12544311]

5. Nickel JC, McNaughton-Collins M, Litwin SM. Use of a validated outcome measure for prostatitis. J Clin Outcomes Manag 2001;8:30.

6. Shoskes DA, Hakim L, Ghoniem G, Jackson CL. Long-term results of multimodal therapy for chronic prostatitis/chronic pelvic pain syndrome. J Urol 2003;169:1406-10. [PubMed: 12629373]

7. Nickel JC. The three As of chronic prostatitis therapy: antibiotics, alpha-blockers and antiinflammatories. What is the evidence. BJU Int 2004;94:1230-3. [PubMed: 15610095]

8. Nickel JC. Chronic prostatitis/chronic pelvic pain syndrome: a decade of change. AUA Update Series 2006;25:309-16.

9. Dimitrakov JD, Kaplan SA, Kroenke K, Jackson JL, Freeman MR. Management of chronic prostatitis/ chronic pelvic pain syndrome: an evidence-based approach. Urology 2006;67:881-8. [PubMed: 16698346] 
10. Shaeffer, AJ.; Anderson, RU.; Krieger, JN., et al. The assessment and management of male pelvic pain syndrome, including prostatitis. In: McConnell, J.; Abrams, P.; Denis, L.; Khoury, S.;

Roehrborn, C., editors. Male lower urinary tract dysfunction, evaluation and management. Heath Publications; Paris: 2006. p. 341-85.

11. Nickel JC, Downey J, Clark J, et al. Levofloxacin for chronic prostatitis/chronic pelvic pain syndrome in men: a randomized placebo-controlled multicenter trial. Urology 2003;62:614-7. [PubMed: 14550427]

12. Cheah PY, Liong ML, Yuen KH, et al. Terazosin therapy for chronic prostatitis/chronic pelvic pain syndrome: a randomized, placebo controlled trial. J Urol 2003;169:592-6. [PubMed: 12544314]

13. Mehik A, Alas P, Nickel JC, Sarpola A, Helström PJ. Alfuzosin treatment for chronic prostatitis/ chronic pelvic pain syndrome: a prospective, randomized, double-blind, placebo controlled, pilot study. Urology 2003;62:425-9. [PubMed: 12946740]

14. Nickel JC, Narayan P, MacKay J, Doyle C. Treatment of chronic prostatitis/chronic pelvic pain syndrome with tamsulosin: a randomized double blind trial. J Urol 2004;171:1594-7. [PubMed: 15017228]

15. Alexander RB, Propert KJ, Schaeffer AJ, et al. Chronic Prostatitis Collaborative Research Network. Ciprofloxacin or tamsulosin in men with chronic prostatitis/chronic pelvic pain syndrome: a randomized, double-blind trial. Ann Intern Med 2004;141:581-9. [PubMed: 15492337]

16. Nickel JC, Pontari M, Moon T, et al. A randomized, placebo controlled, multicenter study to evaluate the safety and efficacy of rofecoxib in the treatment of chronic nonbacterial prostatitis. J Urol 2003;169:1401-5. [PubMed: 12629372]

17. Nickel JC, Forrest JB, Tomera K, et al. Pentosan polysulfate sodium therapy for men with chronic pelvic pain syndrome: a multicenter, randomized, placebo controlled study. J Urol 2005;173:12525. [PubMed: 15758763]

18. Nickel JC, Downey J, Pontari MA, Shoskes DA, Zeitlin SI. A randomized placebo-controlled multicentre study to evaluate the safety and efficacy of finasteride for male chronic pelvic pain syndrome (category IIIA chronic nonbacterial prostatitis). BJU Int 2004;93:991-5. [PubMed: 15142149]

19. De Rose AF, Gallo F, Giglio M, Carmignani G. Role of mepartricin in category III chronic nonbacterial prostatitis/chronic pelvic pain syndrome: a randomized prospective placebo-controlled trial. Urology 2004;63:13-6. [PubMed: 14751338]

20. Shoskes DA, Zeitlin SI, Shahed A, Rajfer J. Quercetin in men with category III chronic prostatitis: a preliminary prospective, double-blind, placebo-controlled trial. Urology 1999;54:960-3. [PubMed: 10604689]

21. Nickel JC, Downey J, Johnston B, Clark J. Canadian Prostatitis Research Group. Predictors of patient response to antibiotic therapy for chronic prostatitis/chronic pelvic pain syndrome: a prospective multicenter clinical trial. J Urol 2001;165:1539-44. [PubMed: 11342913]

22. Nickel JC, Zadeikis N, Spivey M, et al. Clinical significance of antimicrobial therapy in chronic prostatitis associated with non-traditional uropathogens. J Urol 2005;173:S30.

23. Goldmeier D, Madden P, McKenna M, Tamm N. Treatment of category III A prostatitis with zafirlukast: a randomized controlled feasibility study. Int J STD AIDS 2005;16:196-200. [PubMed: 15829018]

24. Reissigl A, Djavan B, Pointner J. Prospective placebo-controlled multicenter trial on safety and efficacy of phytotherapy in the treatment of chronic prostatitis/chronic pelvic pain syndrome. J Urol 2004;171:61. [PubMed: 14665844]Abstract 233

25. Schneider H, Ludwig M, Horstmann A, Schnitker J, Weidner W. The efficacy of Cernilton ${ }^{\circledR}$ in patients with chronic pelvic pain syndrome (CP/CPPS) type NIH IIIa: a randomized, prospective, double-blind, placebo controlled study. J Urol 2006;175

26. Elist J. Effects of pollen extract preparation Prostat/Poltit on lower urinary symptoms in patients with chronic nonbacterial prostatitis/chronic pelvic pain syndrome: a randomized, double-blind, placebocontrolled study. Urology 2006;67:60-3. [PubMed: 16413333]

27. Nickel JC. Opioids for chronic prostatitis and interstitial cystitis: lessons learned from the 11th World Congress on Pain. Urology 2006;68:697-701. [PubMed: 17070334] 
28. Kaplan SA, Te AE, Jacobs BZ. Urodynamic evidence of vesical neck obstruction in men with misdiagnosed chronic nonbacterial prostatitis and the therapeutic role of endoscopic incision of the bladder neck. J Urol 1994;152:2063-5. [PubMed: 7966675]

29. Nickel JC, Sorensen R. Transurethral microwave thermotherapy for nonbacterial prostatitis: a randomized double-blind sham controlled study using new prostatitis specific assessment questionnaires. J Urol 1996;155:1950-4. [PubMed: 8618295]discussion 1954-5

30. Kastner C, Hochreiter W, Huidobro C, Casezas J, Miller P. Cooled transurethral microwave thermotherapy for intractable chronic prostatitis - results of a pilot study after 1 year. Urology 2004;64:1149-54. [PubMed: 15596188]

31. Leskinen MJ, Kilponen A, Lukkarinen O, Tammela TL. Transurethral needle ablation for the treatment of chronic pelvic pain syndrome (category III prostatitis): a randomized, sham-controlled study. Urology 2002;60:300-4. [PubMed: 12137830]

32. Nickel JC, Berger R, Pontari M. Changing paradigms for chronic pelvic pain: a report from The Chronic Pelvic Pain/Chronic Prostatitis Scientific Workshop, October 19-21, 2005, Baltimore, MD. Rev Urol 2006;8:28-35. [PubMed: 16985558] 

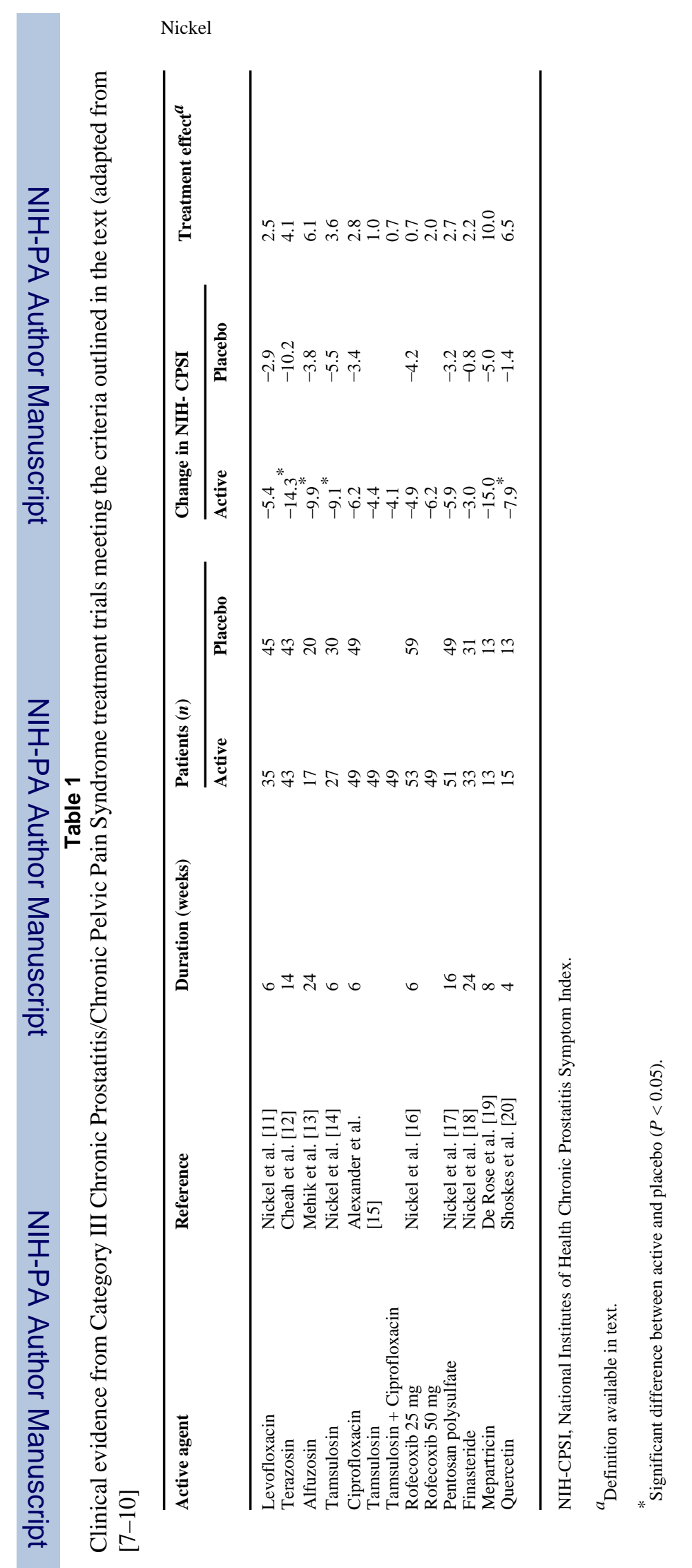

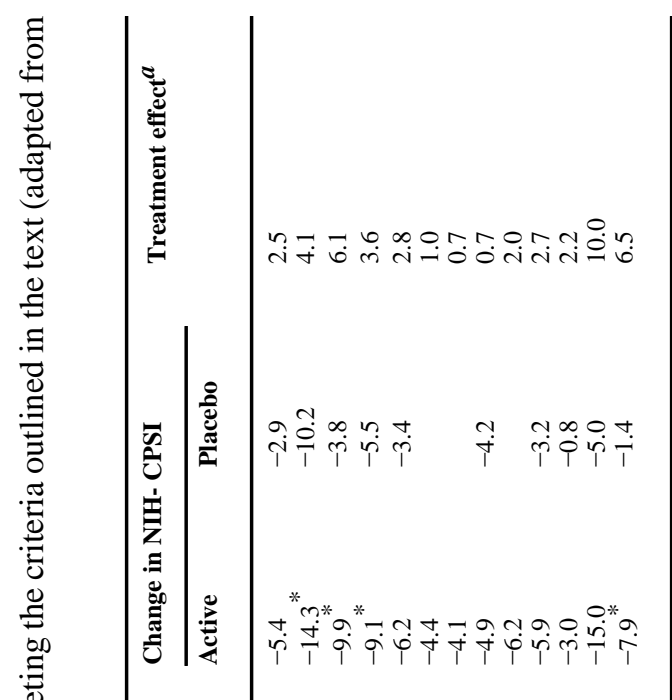

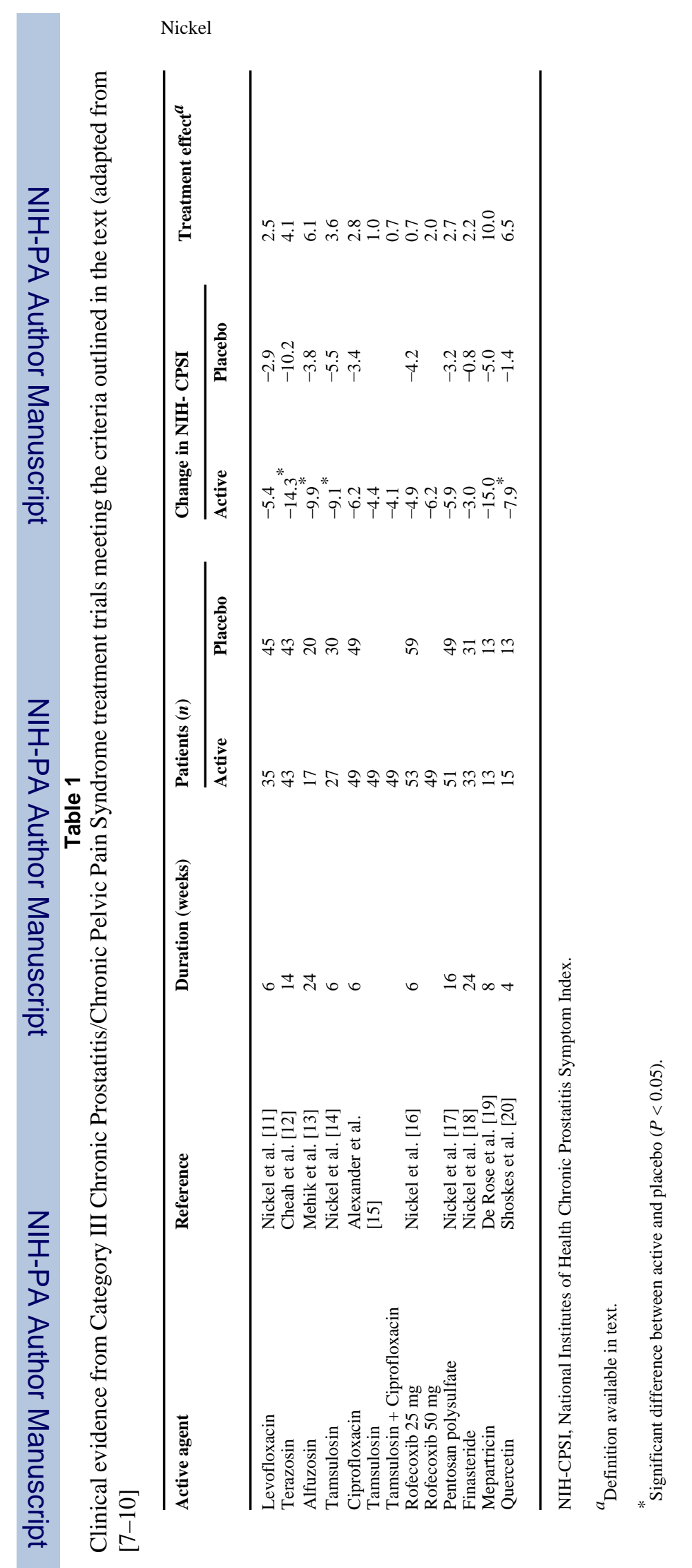

Z

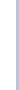

Int J Antimicrob Agents. Author manuscript; available in PMC 2009 February 1. 
Table 2

Recommendations for treatment of chronic prostatitis/chronic pelvic pain syndrome (National Institutes of Health Category III) (adapted from [7-10]

1 Recommended therapies
a. Alpha-blocker therapy for newly diagnosed, alpha-blocker-naïve patients
b. Antibiotics for newly diagnosed, antimicrobial-naïve patients
c. Multimodal therapy

2 Therapies not recommended
a. Alpha-blockers in chronic, heavily pre-treated patients
b. Anti-inflammatory monotherapy
c. Antibiotics in chronic, heavily pre-treated patients
d. 5-alpha reductase inhibitor therapy
e. Minimally invasive therapies
f. Invasive surgical therapies such as prostatectomy

3 Therapies requiring further evaluation
a. Heat therapy
b. Mepartricin
c. Quercetin and cernilton (and other phytotherapies)
d. Biofeedback
e. Physical therapy
f. Acupuncture
g. Electromagnetic stimulation
h. Immunomodulating agents
i. Muscle relaxants
j. Neuromodulating agents
k. Pudendal nerve modulation 